\title{
INEQUALITIES FOR UNITARILY INVARIANT NORMS
}

\section{LIMIN ZOU AND YOUYI JIANG}

Abstract. This paper aims to discuss some inequalities for unitarily invariant norms. We obtain several inequalities for unitarily invariant norms.

Mathematics subject classification (2010): 15A18, 15A42, 15A60.

Keywords and phrases: Unitarily invariant norms, positive semidefinite matrices, inequalities.

\section{REFERENCES}

[1] R. BhatiA, C. DAVIS, More matrix forms of the arithmetic-geometric mean inequality, SIAM J. Matrix Anal. Appl. 14(1993) 132-136.

[2] F. Kittaneh, Y. Manasrah, Improved Young and Heinz inequalities for matrices, J. Math. Anal. Appl. 361(2010) 262-269.

[3] R. Bhatia, F. Kittaneh, Notes on matrix arithmetic-geometric mean inequalities, Linear Algebra Appl. 308(2000) 203-211.

[4] Y. TAO, More results on singular value inequalities of matrices, Linear Algebra Appl. 416(2006) 724 729.

[5] R. Bhatia, Matrix Analysis, Springer-Verlag, New York, 1997.

[6] R. A. Horn, X. Zhan, Inequalities for C-S seminorms and Lieb functions, Linear Algebra Appl. 291(2000) 103-113.

[7] H. Kosaki, Arithmetic-geometric mean and related inequalities for operators, J. Funct. Anal. 156(1998) 429-451.

[8] F. HIAI, X. ZHAN, Inequalities involving unitarily invariant norms and operator monotone functions, Linear Algebra Appl. 341(2002) 151-169. 